\title{
Otimismo e suporte social em mulheres com câncer de mama: uma revisão sistemática
}

\author{
Micheline Roat Bastianello' \\ Universidade Federal Fluminense, Niterói - RJ - Brasil \\ Claudio Simon Hutz \\ Universidade Federal do Rio Grande do Sul, Porto Alegre - RS - Brasil
}

\begin{abstract}
Resumo: Este trabalho teve como objetivo realizar uma revisão sistemática da literatura científica sobre as relações entre otimismo e suporte social em mulheres com câncer de mama. As buscas eletrônicas foram executadas a partir da utilização dos descritores "otimismo" (optimism), "suporte social" (social support) e "câncer de mama" (breast cancer) e do operador booleano "e" (and). Pesquisou-se nas bases de dados: PsycINFO, Scopus, Sage Publications, SciELO, Medline/PubMed Resources Guide. Buscaram-se artigos publicados entre os anos 1994-20I4. Os artigos foram analisados a partir de cinco dimensões: objetivo, instrumentos, amostra, análise dos dados e principais resultados. Observou-se que a produção científica sobre a temática estudada é pequena e apresenta um crescimento lento. Com relação aos instrumentos de coleta de dados, a escala mais utilizada para medir otimismo foi a Revised Life Orientation Test (LOT-R), enquanto para medir suporte social um número variado de escalas foi utilizado. Os resultados sugerem que as mulheres com maior otimismo e suporte social tendem a se envolver em comportamentos mais saudáveis, contribuindo para melhor qualidade de vida.
\end{abstract}

Palavras-chave: otimismo; suporte social; câncer de mama; psicologia positiva, revisão de literatura.

\section{OPTIMISM AND SOCIAL SUPPORT IN WOMEN WITH BREAST CANCER: A SYSTEMATIC REVIEW}

\begin{abstract}
The present study aimed to conduct a systematic review of the scientific literature on the relation between optimism and social support in women with breast cancer. Electronic searches were performed using the descriptors optimism, social support, and breast cancer in English and Portuguese with the Boolean operator "and". The following data bases were used: PsycINFO, Scopus, Sage Publications, SciELO, Medline/PubMed Resources Guide. The study included papers published between the years 1994-2014. The articles were analyzed using five dimensions: objectives instruments sample analysis of the data and main results. The results showed that the scientific literature on the subject studied is still scarce and shows a slow growth. Regarding data collection instruments, the most widely used scale to measure optimism was the Revised Life Orientation Test (LOT-R), while to measure social support several different scales were used. The results suggest that women with greater optimism and social support tend to engage in healthier behaviors, contributing to a better quality of life.
\end{abstract}

Keywords: optimism; social support; breast cancer; positive psychology; literature review.

${ }^{1}$ Endereço para correspondência: Micheline Roat Bastianello, Universidade Federal Fluminense, Rua José do Patrocínio, 71, Centro - Campo dos Goytacazes - RJ - Brasil. CEP: 28010-385. E-mail: mbastianello@hotmail.com. 
OPTIMISMO Y APOYO SOCIAL EN MUJERES CON CÁNCER DE MAMA: UNA REVISIÓN SISTEMÁTICA

\begin{abstract}
Resumen: Este estudio tiene como objetivo llevar a cabo una revisión sistemática de la literatura científica sobre la relación entre el optimismo y el apoyo social en las mujeres con cáncer de mama. Las búsquedas electrónicas se realizaron entre I994-20I 4 y utilizado las palabras "optimismo", "apoyo social" y "cáncer de mama" y el operador booleano " $y$ " en portugués e inglés. Las bases de datos busquedo: PsycINFO, Scopus, Sage Publications, SciELO, Medline/PubMed Resources Guide. Los artículos fueron analizados a partir de cinco dimensiones: propósito, instrumentos, muestras, análisis de datos y resultados principales. Se observó que la literatura científica sobre el tema estudiado es pequeña y tiene un crecimiento lento. Con relación a los instrumentos de recolección de datos, la escala más utilizada para medir el optimismo fue el Life Orientation Test Revisado (LOT-R), mientras que para medir el apoyo social se utilizó una serie de diferentes escalas. Los resultados sugieren que las mujeres con mayor optimismo y el apoyo social presentan comportamientos más saludables, lo que contribuye a una mejor calidad de vida.
\end{abstract}

Palabras clave: optimismo; apoyo social; cáncer de mama; psicología positiva; revisión de literatura.

Este trabalho teve como objetivo realizar uma revisão sistemática da literatura científica sobre as relações entre otimismo e suporte social em mulheres com câncer de mama a partir da perspectiva da psicologia positiva. O câncer de mama é o segundo tipo de neoplasia mais frequente em mulheres e se destaca como a terceira causa de morte entre brasileiras adultas. No Brasil, a estimativa para 2016 é de aproximadamente 57.960 novos casos de CA de mama, com um risco estimado de 56,09 casos a cada 100 mil mulheres. Esse tipo de câncer é o mais frequente nas mulheres das regiões Sudeste (71,18/100 mil) e Sul (70,98/ 100 mil) (INCA/2016).

Diante de tal quadro, pesquisas sobre os aspectos psicológicos do câncer de mama vêm sendo realizadas. A maior parte delas tem como tema o estresse, a ansiedade, a depressão e o pessimismo advindos da experiência de diagnóstico e tratamento da doença (Zenger, Glaesmer, Hoöckel, \& Hinz, 2011). Também são assuntos recorrentes as repercussões psicológicas nas diferentes fases do adoecimento e tratamento (pré-diagnóstico, diagnóstico, tratamento e pós-tratamento), o ajustamento psicossocial e a baixa qualidade de vida da mulher acometida por câncer de mama (Rossi \& Santos, 2003; Silva, 2008).

Contudo, pesquisas sobre questões relacionadas às forças de caráter, às emoções e aos vínculos positivos, como otimismo e suporte social, parecem ser pouco abordadas. Quando as pessoas passam por dificuldades ou desafios em suas vidas experienciam uma grande variedade de emoções negativas e positivas. Estudos indicam que o balanço entre tais emoções parece estar relacionado com o grau de otimismo e pessimismo das pessoas (Scheier, Carver, \& Bridges, 2001). Segundo Scheier e Carver (1985), otimistas são pessoas que esperam que boas coisas aconteçam, mesmo quando existem dificuldades ou o cenário não se apresenta como favorável. Este senso de confiança, característico dos indivíduos otimistas, produz um misto de emoções positivas, como tranquilidade, compaixão, alegria, bem-estar.

As características das pessoas otimistas têm chamado atenção de pesquisadores nos ambientes médicos, e diversas pesquisas têm sido conduzidas com o objetivo de 
investigar o papel do otimismo no enfrentamento e tratamento de doenças. Evidências empíricas demonstram que otimismo está associado à melhor recuperação de cirurgias coronarianas (Fitzgerald, Tennen, Affleck, \& Pransky, 1993) e transplantes de medula óssea (Curbow, Somerfield, Baker, Wingard, \& Legro, 1993), assim como melhor enfrentamento de doenças como câncer (Carver, Pozo, Harris, Noriega, Scheier, \& Robinson, 1993) e AIDS (Taylor, Kemeny, Aspinwall, Schneider, Rodriguez, \& Herbert, 1992).

Pesquisadores também descobriram que pessoas otimistas estão mais engajadas na promoção da saúde no sentido que parecem tomar as medidas necessárias para minimizar os riscos relativos às desordens ou patologias, como comportamentos de risco para ataques cardíacos (Radcliffe \& Klein, 2002), para contaminação pelo vírus HIV (Taylor et al., 1992) e para alcoolismo (Ohannessian, Hesselbrock, Tennen, \& Affleck, 1994). O otimismo pode ser entendido como uma estratégia comportamental que permite às pessoas terem mais saúde física e mental, assim como engajarem-se em atividades para sua manutenção (Shane \& Snyder, 2003).

Somando-se ao otimismo, tem chamado à atenção dos cientistas a importância das relações sociais durante o tratamento de doenças e na manutenção do bem-estar e qualidade de vida do paciente. Um aspecto das relações sociais que vem sendo estudado desde a década de 1970 é o suporte social.

Tem sido demonstrado que o suporte social reduz os efeitos negativos do estresse advindo do adoecimento sobre a saúde, uma vez que auxilia na promoção de estratégias de enfrentamento (Nurullah, 2012). Pesquisadores notaram que portadores de enfermidades diversas mais expostos ao estresse e a ansiedade relatam com frequência a ausência de uma rede social (Chor, Griep, Lopes, \& Faerstein, 2001). Por outro lado, pessoas que percebem ter suporte social apresentam melhor ajustamento emocional, recuperam-se mais rapidamente de situações traumáticas e apresentam maior sobrevida no decurso de doenças crônicas (Cohen, Underwood, \& Gottlieb, 2000).

Em face dessas considerações traçou-se o objetivo deste trabalho, a saber: examinar estudos que vinculem otimismo e suporte social em mulheres com CA de mama.

\section{Método}

\section{Estratégias de busca das referências}

O material necessário para a revisão sistemática da literatura científica foi obtido mediante buscas em diferentes bases de dados eletrônicas. Optou-se por bases mais relevantes para a área da Psicologia. Foram consultadas as seguintes bases de dados: PsycINFO, Scopus, Sage Publications, SciELO, Medline/PubMed Resources Guide.

As buscas eletrônicas foram executadas a partir da utilização dos descritores "otimismo" (optimism), "suporte social" (social support) e "câncer de mama" (breast cancer) e do operador booleano "e" (and). Essa estratégia foi adotada porque viabiliza a 
localização de referências que apresentam os três descritores conjuntamente. As bases de dados foram configuradas para localizar as referências que apresentavam os descritores no "assunto", "título" e "resumo".

Foi delimitado o espaço de tempo entre 1994 e 2014 para a localização das referências. Foram selecionados os últimos 20 anos por ser um período característico da expansão dos estudos sobre bem-estar subjetivo e emoções positivas, como o otimismo (Pureza, Kuhn, Castro, \& Lisboa, 2012; Pacico \& Bastianello, 2014).

O processo de localização e seleção dos artigos foi realizado em três etapas. A primeira etapa consistiu na leitura dos títulos dos artigos que foram localizados nas buscas, a fim de excluir aqueles que claramente não atendiam aos critérios de inclusão previamente estabelecidos, os quais serão apresentados a seguir. Na segunda etapa, este mesmo procedimento foi efetuado, sendo que agora a decisão pela exclusão foi baseada nas informações constantes dos resumos. Os resumos que não atenderam os critérios de inclusão foram descartados. Na última etapa, os artigos foram analisados na íntegra.

Nas três etapas de seleção e análise dos artigos, o processo foi conduzido de modo independente por dois juízes. A seleção foi conduzida, no mês de agosto de 2014, durante o mesmo intervalo de tempo concomitantemente por ambos os juízes com a finalidade de minimizar o viés no processo. Depois de cada etapa, os juízes confrontavam as análises efetuadas e, na ocorrência de divergência, os dois, conjuntamente, efetuavam novamente a leitura do resumo e, se necessário, do artigo, a fim de decidir consensualmente pela inclusão.

\section{Procedimentos para seleção e apreciação das referências}

Os critérios de inclusão estabelecidos foram: 1. idioma: referências escritas em língua inglesa, espanhola e portuguesa; 2. formato: referências publicadas como artigo, relato de experiência ou revisão da literatura científica; 3 . objetivo: referências voltadas especificamente ao estudo do otimismo e suporte social em mulheres acometidas por câncer de mama.

As referências selecionadas foram submetidas a uma apreciação qualitativa com o auxílio dos critérios de inclusão supracitados e da leitura analítica de cada uma delas, levando-se em consideração características, como: autoria, ano, periódico de publicação, país do estudo, tamanho da amostra e idade média das participantes. Os achados dessas leituras foram analisados desde cinco dimensões de análise: objetivo, instrumentos, amostra, análise dos dados e principais resultados.

\section{Resultados}

\section{Apreciação quantitativa das referências}

As buscas eletrônicas possibilitaram a localização de 69 referências potencialmente relevantes. A maior parte delas foi encontrada nas bases Psylnfo (20 artigos) e Medli- 
ne/PubMed (40 artigos), como demonstra a Figura 1. Os artigos duplicados foram removidos restando 44 referências. Os resumos dessas referências foram submetidos aos critérios de inclusão estabelecidos e restaram sete artigos para análise final.

Os sete artigos selecionados foram publicados entre 2003 e 2011 . A metade deles foi publicada a partir de 2008, aspecto que confirma a hipótese inicial deste estudo sobre a atualidade dos temas investigados. Na Figura 1, os artigos estão caracterizados por autoria, ano de publicação do artigo, nome do periódico de publicação, país do estudo, tamanho da amostra e idade média das participantes.

\section{Figura I. Resultado da busca nas bases de dados eletrônicas}

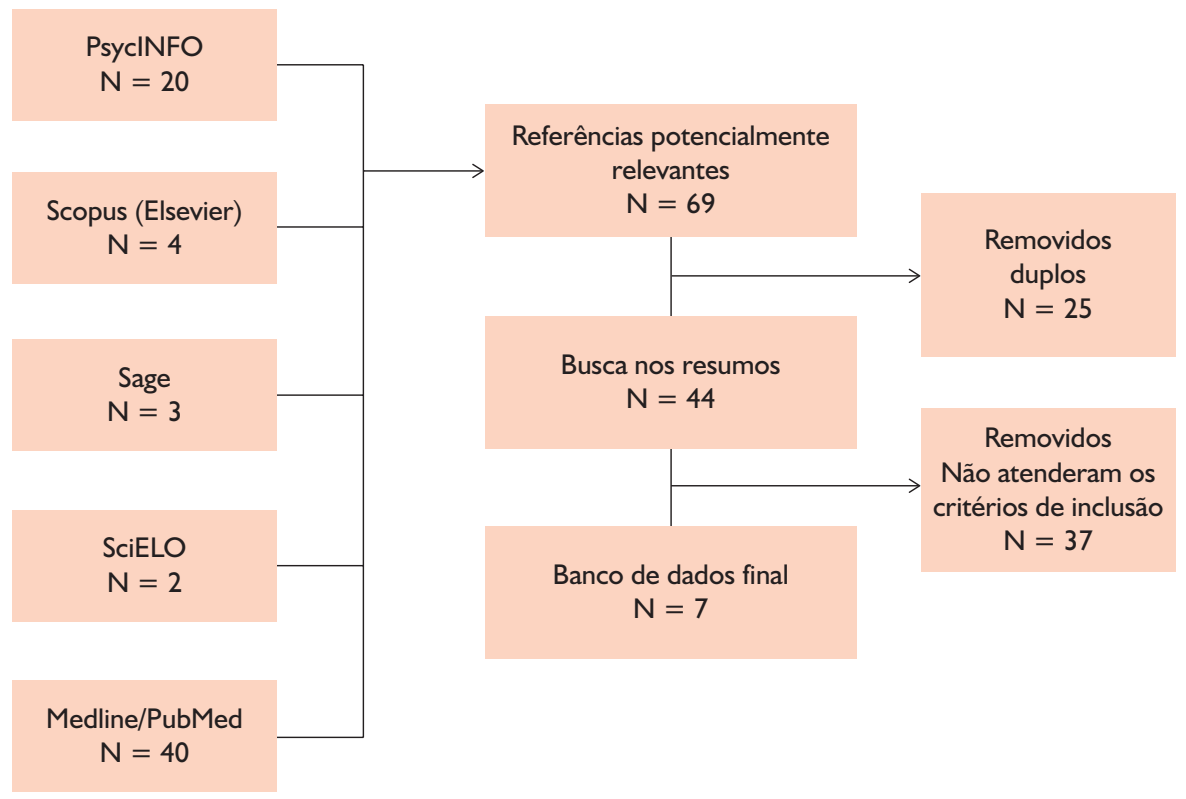

Fonte: Elaborada pelos autores.

\section{Apreciação qualitativa das referências}

As características dos estudos (autores, ano da publicação, periódico, país de realização, tamanho e idade média da amostra) são apresentadas na Tabela 1. Os estudos 2, 4, 5 e 6 adotaram delineamento transversal, enquanto os estudos 1, 3 e 7 foram longitudinais.

O periódico Psycho-Oncology apresentou dois estudos, enquanto os demais apenas um. Observa-se que dos sete estudos apenas um não teve como país de origem os Estados Unidos. Com relação ao número de participantes, as amostras variaram entre 44 a 148 mulheres com câncer de mama. A idade média das mulheres variou de 46,28 a 59,7 . 
Tabela I. Principais características dos estudos incluídos na revisão

\begin{tabular}{|c|c|c|c|c|c|}
\hline Autor & Ano & Periódico & $\begin{array}{l}\text { País do } \\
\text { estudo }\end{array}$ & $\begin{array}{l}\text { Tamanho } \\
\text { da } \\
\text { amostra }\end{array}$ & $\begin{array}{l}\text { Idade média } \\
\text { M(DP) }\end{array}$ \\
\hline I. Trunzo, J. J., \& Pinto, B. M. & 2003 & $\begin{array}{l}\text { Journal of Consulting } \\
\text { and Clinical Psychology }\end{array}$ & Estados Unidos & 69 & $57,5(13,2)$ \\
\hline $\begin{array}{l}\text { 2. Von Ah, D., Kang, D. H., \& } \\
\text { Carpenter, J. S. }\end{array}$ & 2007 & $\begin{array}{l}\text { Research in Nursing } \\
\text { \& Healt }\end{array}$ & Estados Unidos & 54 & $51,9(9,6)$ \\
\hline $\begin{array}{l}\text { 3. Shelby, R. A., Crespin, T. R., } \\
\text { Wells-Di Gregorio, S. M., Lamdan, } \\
\text { R. M., Siegel, J. E., \& Taylor, K. L. }\end{array}$ & 2008 & $\begin{array}{l}\text { Journal of Behavioral } \\
\text { Medicine }\end{array}$ & Estados Unidos & 148 & $53,5(12,8)$ \\
\hline $\begin{array}{l}\text { 4. Wimberly, S. R., Carver, C. S., } \\
\text { \& Antoni, M. H. }\end{array}$ & 2008 & Psychologyand Health & Estados Unidos & 136 & $50,25(9,15)$ \\
\hline $\begin{array}{l}\text { 5. Bozo, Ö. Z., GündogDu, E., \& } \\
\text { Ik-Çolak, C. B. }\end{array}$ & 2009 & $\begin{array}{l}\text { Journal of Healt } \\
\text { Psychology }\end{array}$ & Turquia & 104 & $46,28(9,23)$ \\
\hline 6. Matthews, E. E., \& Cook, P. F. & 2009 & Psychooncology & Estados Unidos & 93 & $59,7(I I, 2)$ \\
\hline $\begin{array}{l}\text { 7. Smith, S. K., Hemdon, J. E., } \\
\text { Lyerly, H. K., Coan, A., Wheeler, } \\
\text { J. L., Staley, T., \& Abernethy, A. P. }\end{array}$ & 2011 & Psychooncology & Estados Unidos & 44 & $5 I, 5(I I, 9)$ \\
\hline
\end{tabular}

Fonte: Elaborada pelos autores.

O Quadro 1 apresenta os objetivos, amostras e instrumentos presentes em cada um dos sete estudos selecionados. Os estudos 1 e 4 tiveram como objetivo examinar a relação do otimismo com bem-estar (BES) psicossexual e sofrimento emocional, tendo como mediador o suporte social. Nos estudos 3 e 5, o suporte social aparece como variável moderadora das relações de otimismo com ajustamento e crescimento póstraumático. Em um único estudo (2) o otimismo foi avaliado como moderador dos efeitos do estresse sobre as respostas imunes das pacientes. No estudo 6 , suporte social, coping e autotranscendência foram testados como mediadores do otimismo para o bem-estar. E o estudo 7 avaliou a correlação entre os recursos psicossociais, como otimismo, suporte social, autoeficácia, enfrentamento e espiritualidade, alvos de um programa de saúde, e os benefícios experimentados pelas pacientes.

As participantes de todos os estudos foram mulheres com câncer de mama que atendiam a pelo menos um critério preestabelecido (câncer não ser metastático, se encontrar em pós-operatório, estar em tratamento com radioterapia etc.). O único estudo que apresentou uma lista de oito critérios para as pacientes serem elegíveis foi o 7 .

Com relação aos instrumentos utilizados para a coleta de dados, observa-se que todos os estudos utilizaram para medir otimismo a escala Life Orientation Test Revised 
(LOT-R; Scheier, Carver, \& Bridges, 1994), sendo que apenas os estudos 3 e 6 utilizaram a primeira versão dessa escala, a LOT (Scheier \& Carver, 1985). Entretanto, para medir suporte social, a maior parte dos estudos utilizou uma escala distinta. O estudo 2 utilizou a SSQ-6, que é uma versão adaptada da SSQ empregada no estudo 6 . E o estudo 3 trabalhou com a ISEL-SF, que é a versão curta da ISEL usada no estudo 7. Os demais estudos usaram escalas diferentes, como pode ser visto a seguir.

\section{Quadro I. Descrição dos objetivos, amostras e instrumentos dos artigos selecionados}

\begin{tabular}{|c|c|c|c|}
\hline Título do artigo & Objetivo & Amostra & Instrumentos \\
\hline $\begin{array}{l}\text { I. Social Support } \\
\text { as a Mediator of } \\
\text { Optimism and } \\
\text { Distress } \\
\text { in Breast Cancer } \\
\text { Survivors }\end{array}$ & $\begin{array}{l}\text { Este estudo investigou como } \\
\text { o suporte social (afetivo } \\
\text { e confidente) afeta a relação } \\
\text { entre otimismo e sofrimento } \\
\text { emocional. Foi testada } \\
\text { a hipótese de que o suporte } \\
\text { social (variável mediadora) } \\
\text { tem um efeito de mediação } \\
\text { no otimismo (preditor) } \\
\text { e sofrimento emocional } \\
\text { (variável critério). }\end{array}$ & $\begin{array}{l}\text { Mulheres em estágio } \\
\text { inicial de câncer } \\
\text { de mama }\end{array}$ & $\begin{array}{l}\text { I. Duke-UNC Functional Social } \\
\text { Support Scale } \\
\text { (Broadhead et al., 1988) } \\
\text { 2. Life Orientation Test-Revised } \\
\text { (LOT-R; Scheier, Carver, \& } \\
\text { Bridges, I994) } \\
\text { 3. Profile of Mood States-Short } \\
\text { Form (POMS-SF; McNair, Lorr, } \\
\text { \& Droppelman, 1992) }\end{array}$ \\
\hline $\begin{array}{l}\text { 2. Stress, Optimism, } \\
\text { and Social Support: } \\
\text { Impact on Immune } \\
\text { Responses in Breast } \\
\text { Cancer }\end{array}$ & $\begin{array}{l}\text { O objetivo deste estudo foi } \\
\text { examinar o efeito direto } \\
\text { do otimismo e satisfação } \\
\text { de vida com o suporte social } \\
\text { sobre as respostas imunes } \\
\text { de mulheres com câncer } \\
\text { de mama. }\end{array}$ & $\begin{array}{l}\text { Mulheres com câncer } \\
\text { de mama no pós- } \\
\text { operatório }\end{array}$ & $\begin{array}{l}\text { I. Event Scale (IES, Horowitz, } \\
\text { Wilner, \& Alvarez, I979) } \\
\text { 2. Life Orientation Test-Revised } \\
\text { (LOT-R; Scheier, Carver, } \\
\text { \& Bridges, I994) } \\
\text { 3. Social Support Questionnaire } \\
\text { (SSQ-6; I. G. Sarason, I987) } \\
\text { 4. Immunological assessments: } \\
\text { NKCA e Cytokine level: } \\
\text { IFN-c }\end{array}$ \\
\hline $\begin{array}{l}\text { 3. Optimism, social } \\
\text { support, and } \\
\text { adjustment in } \\
\text { African American } \\
\text { women with breast } \\
\text { cancer }\end{array}$ & $\begin{array}{l}\text { O objetivo deste estudo foi } \\
\text { examinar as relações entre } \\
\text { otimismo, apoio social } \\
\text { e ajustamento em uma } \\
\text { amostra de mulheres afro- } \\
\text { americanas com CA de } \\
\text { mama que participam de um } \\
\text { grupo de apoio psicossocial. }\end{array}$ & $\begin{array}{l}\text { Mulheres afro- } \\
\text { americanas com } \\
\text { câncer de mama não } \\
\text { metastático }\end{array}$ & $\begin{array}{l}\text { I. Life orientation test (LOT) } \\
\text { (Scheier \& Carver, I985) } \\
\text { 2. Interpersonal Support } \\
\text { Evaluations list - short form } \\
\text { (ISEL-SF) } \\
\text { 3. Mental health inventory (MHI) } \\
\text { 4. Cancer rehabilitation } \\
\text { evaluation system - short form } \\
\text { (CARES-SF) }\end{array}$ \\
\hline
\end{tabular}




\section{Quadro I. Descrição dos objetivos, amostras e instrumentos dos artigos selecionados (continuação)}

\begin{tabular}{|c|c|c|c|}
\hline Título do artigo & Objetivo & Amostra & Instrumentos \\
\hline $\begin{array}{l}\text { 4. Effects of } \\
\text { optimism, } \\
\text { interpersonal } \\
\text { relationships, } \\
\text { and distress on } \\
\text { psychosexual well- } \\
\text { being among women } \\
\text { with early stage } \\
\text { breast cancer }\end{array}$ & $\begin{array}{l}\text { Examinar o suporte social } \\
\text { emocional como mediador } \\
\text { potencial do efeito de } \\
\text { otimismo sobre o } \\
\text { bem-estar psicossexual. }\end{array}$ & $\begin{array}{l}\text { Mulheres nos estádios } \\
0, \text { I e II de câncer } \\
\text { de mama }\end{array}$ & $\begin{array}{l}\text { I. Life Orientation Test-Revised } \\
\text { (LOT-R; Scheier, Carver, } \\
\text { \& Bridges, I994) } \\
\text { 2. Social Provisions Scale (SPS; } \\
\text { Cutrona \& Russell, I987) } \\
\text { 3. Emotional distress - } \\
\text { It measures general mood } \\
\text { disturbance with a series } \\
\text { of adjectives, each of which } \\
\text { is a mood descriptor } \\
\text { (Carver et al., I993) } \\
\text { 4. Psychosexual well-being. } \\
\text { (Carver et al., I998) }\end{array}$ \\
\hline $\begin{array}{l}\text { 5. The Moderating } \\
\text { Role of Different } \\
\text { Sources of } \\
\text { Perceived Social } \\
\text { Support on the } \\
\text { Dispositional } \\
\text { Optimism } \\
\text { Posttraumatic } \\
\text { Growth } \\
\text { Relationship in } \\
\text { Postoperative } \\
\text { Breast } \\
\text { Cancer Patients }\end{array}$ & $\begin{array}{l}\text { O objetivo do presente } \\
\text { estudo foi examinar } \\
\text { a associação entre } \\
\text { otimismo disposicional } \\
\text { e crescimento pós- } \\
\text { traumático (PTG), } \\
\text { e o efeito moderador } \\
\text { do suporte social nessa } \\
\text { relação para } \\
\text { sobreviventes de câncer } \\
\text { de mama no } \\
\text { pós-operatório. }\end{array}$ & $\begin{array}{l}\text { Mulheres com câncer } \\
\text { de mama em } \\
\text { tratamento } \\
\text { quimioterápico ou } \\
\text { vindo ao hospital para } \\
\text { seus tratamentos } \\
\text { de rotina. }\end{array}$ & $\begin{array}{l}\text { I. Life Orientation Test- } \\
\text { Revised (LOT-R; Scheier, } \\
\text { Carver, \& Bridges, I994) } \\
\text { 2. Multidimensional Scale } \\
\text { of Perceived Social Support } \\
\text { (MSPSS) } \\
\text { 3. Posttraumatic Growth } \\
\text { Inventory (PTGI; Tedeschi } \\
\text { \& Calhoun, I996) }\end{array}$ \\
\hline $\begin{array}{l}\text { 6. Relationships } \\
\text { among optimism, } \\
\text { well-being, self- } \\
\text { transcendence, } \\
\text { coping, and social } \\
\text { support in women } \\
\text { during treatment } \\
\text { for breast } \\
\text { cancer }\end{array}$ & $\begin{array}{l}\text { O objetivo deste estudo } \\
\text { foi investigar a relação } \\
\text { entre otimismo } \\
\text { e bem-estar subjetivo. } \\
\text { E a mediação individual } \\
\text { e combinado dessa } \\
\text { relação pelo suporte } \\
\text { social percebido, coping } \\
\text { e auto-transcendência } \\
\text { em mulheres com câncer } \\
\text { de mama durante } \\
\text { a radioterapia. }\end{array}$ & $\begin{array}{l}\text { Mulheres com câncer } \\
\text { de mama em } \\
\text { tratamento com } \\
\text { radioterapia. }\end{array}$ & $\begin{array}{l}\text { I. The Positive Affect } \\
\text { Scale (PA) } \\
\text { 2. The Life Orientation } \\
\text { Test (LOT) } \\
\text { 3. The Social Support } \\
\text { Questionnaire (SSQ) } \\
\text { 4. The Jalowiec Coping Scale } \\
\text { 5. The I5-item Self } \\
\text { Transcendence } \\
\text { Scale (STS) } \\
\text { 6. The Symptom Distress } \\
\text { Scale (SDS) }\end{array}$ \\
\hline
\end{tabular}


Quadro I. Descrição dos objetivos, amostras e instrumentos dos artigos selecionados (conclusão)

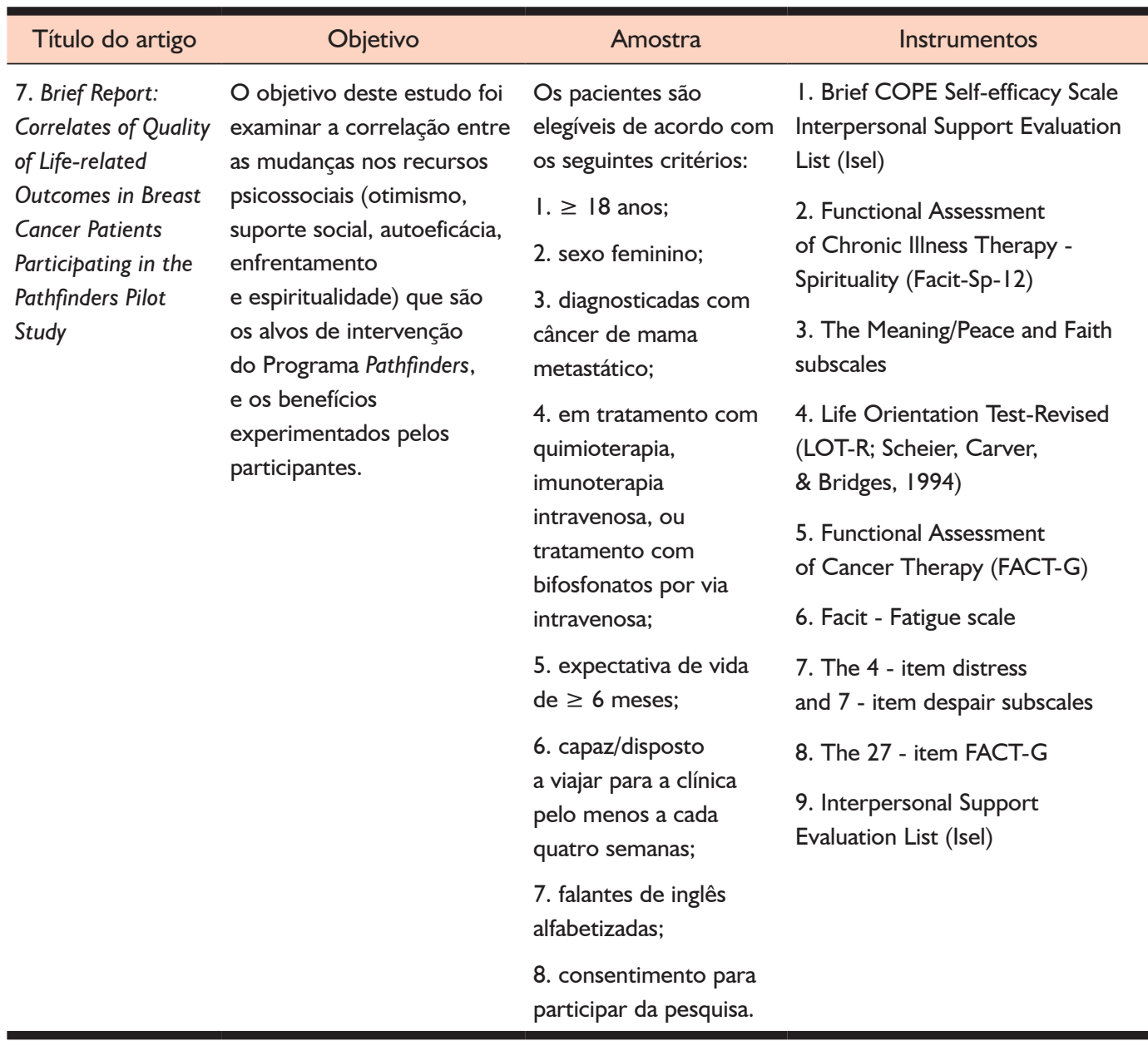

Fonte: Elaborado pelos autores.

Os métodos de análise de dados e os principais resultados de cada artigo são apresentados no Quadro 2. Todos os estudos utilizaram como método de análise modelos de regressão e coeficiente de correlação, e um estudo (o de número 1), além desses métodos, foi utilizada a Análise de Variância (Analysis of Variance - ANOVA).

Com relação aos resultados, os seis primeiros estudos apresentaram evidências de algum tipo de associação entre otimismo e suporte social. Nos estudos 1 e 4, confirmou-se a hipótese de que o suporte social é mediador do otimismo em relação ao bem-estar psicossexual e sofrimento emocional. Nos estudos 3 e 5, o suporte social foi moderador das relações de otimismo com ajustamento e crescimento póstraumático. Em um único estudo (o de número 2), o otimismo foi avaliado como moderador dos efeitos do estresse sobre as respostas imunes das pacientes. No estudo 6 , suporte social, coping e autotranscendência foram testados como mediado- 
res do otimismo para o bem-estar. E o estudo 7 avaliou a correlação entre os recursos psicossociais como, otimismo, suporte social, autoeficácia, enfrentamento e espiritualidade, alvos de um programa de saúde, e os benefícios experimentados pelas pacientes.

\section{Quadro 2. Descrição da análise dos dados e principais resultados dos artigos selecionados}

\begin{tabular}{|c|c|c|}
\hline Título do artigo & $\begin{array}{l}\text { Análise dos } \\
\text { dados }\end{array}$ & Principais resultados \\
\hline \multirow{3}{*}{$\begin{array}{l}\text { I. Social Support } \\
\text { as a Mediator } \\
\text { of Optimism and } \\
\text { Distress in Breast } \\
\text { Cancer Survivors }\end{array}$} & $\begin{array}{l}\text { Coeficiente de } \\
\text { correlação de } \\
\text { Pearson }\end{array}$ & $\begin{array}{l}\text { Suporte social emocional é mediador da associação negativa entre } \\
\text { otimismo e sofrimento emocional no diagnóstico precoce de câncer } \\
\text { de mama, e depois de seis meses transcorridos, mas não depois } \\
\text { de um ano. }\end{array}$ \\
\hline & ANOVA & $\begin{array}{l}\text { De acordo com os pesquisadores, algumas questões não ficaram } \\
\text { claras: }\end{array}$ \\
\hline & Regressões & $\begin{array}{l}\text { I. por que o efeito mediador do suporte social emocional não estava } \\
\text { presente depois de um ano; } \\
\text { 2. por que o estágio da doença e o tipo de tratamento relacionaram-se } \\
\text { com sofrimento emocional aos } 12 \text { meses, mas não no início } \\
\text { do tratamento e em } 6 \text { meses; } \\
\text { 3. por que suporte social íntimo não se relacionou significativamente } \\
\text { com otimismo. }\end{array}$ \\
\hline $\begin{array}{l}\text { 2. Stress, Optimism, } \\
\text { and Social } \\
\text { Support: Impact on } \\
\text { Immune }\end{array}$ & $\begin{array}{l}\text { Coeficiente de } \\
\text { correlação de } \\
\text { Pearson }\end{array}$ & $\begin{array}{l}\text { O achado mais robusto foi a relação significativa e negativa entre } \\
\text { estresse e NKCA e IFN-g (índices que medem a imunidade). } \\
\text { O otimismo atuou como moderador nos efeitos do estresse sobre } \\
\text { os níveis de NKCA, mas não sobre os níveis de IFN-g. A consistência }\end{array}$ \\
\hline Responses in Breast & Regressões & dos resultados ressalta o efeito negativo do estresse advindo do câncer \\
\hline Cancer & $\begin{array}{l}\text { Hierárquicas } \\
\text { Múltiplas } \\
\text { (2 steps) }\end{array}$ & $\begin{array}{l}\text { sobre as respostas imunes das mulheres no pós-cirúrgico. A satisfação } \\
\text { com o suporte social não se relaciona com as respostas imunes. } \\
\text { Os resultados sugerem que as intervenções destinadas a reduzir estresse } \\
\text { e aumentar o otimismo em mulheres com câncer de mama podem } \\
\text { promover boas respostas imunes. }\end{array}$ \\
\hline
\end{tabular}

3. Optimism, social Regressões support, and adjustment in

African American women with breast cancer
Em contraste com estudos anteriores, o apoio social não mediou multilineares a relação entre otimismo e ajustamento na amostra de mulheres afroamericanas. Em vez disso, o apoio social foi moderador da relação entre otimismo e ajustamento, funcionando como um tampão do impacto negativo do baixo otimismo sobre o sofrimento psíquico, bem-estar e funcionamento psicossocial. Mulheres com altos níveis de apoio social apresentam melhor ajustamento mesmo quando o otimismo foi baixo. Em contraste, nas mulheres com altos níveis de otimismo, o aumento do apoio social não forneceu um benefício adicional. Estes dados sugerem que $o$ apoio social percebido é um recurso importante para as mulheres com baixo otimismo. 


\section{Quadro 2. Descrição da análise dos dados e principais resultados dos artigos selecionados (conclusão)}

\begin{tabular}{ccc} 
& Análise dos \\
Título do artigo & dados & Principais resultados \\
\hline
\end{tabular}

\begin{tabular}{|c|c|c|}
\hline $\begin{array}{l}\text { 4. Effects of } \\
\text { optimism, } \\
\text { interpersonal } \\
\text { relationships, } \\
\text { and distress on } \\
\text { psychosexual well- } \\
\text { being among } \\
\text { women with early } \\
\text { stage breast cancer }\end{array}$ & $\begin{array}{l}\text { Coeficiente de } \\
\text { correlação de } \\
\text { Pearson } \\
\text { Regressões } \\
\text { Hierárquicas } \\
\text { Múltiplas } \\
\text { (2 steps) }\end{array}$ & $\begin{array}{l}\text { Os resultados confirmam dois modelos de mediação. O primeiro } \\
\text { modelo indica que pacientes mais otimistas experimentem melhor } \\
\text { bem-estar psicossexual (por exemplo, sentem-se mais femininas, } \\
\text { atraentes e sexualmente desejáveis) em parte porque elas percebem } \\
\text { a si mesmas como tendo mais apoio social disponível. O segundo } \\
\text { modelo indica que as pacientes mais otimistas experienciam maior } \\
\text { bem-estar psicossexual, em parte, porque elas apresentam menor } \\
\text { sofrimento emocional relacionado à doença. Quando os dois modelos } \\
\text { foram testados simultaneamente, o sofrimento emocional não contribuiu } \\
\text { unicamente ao modelo em qualquer tempo exceto depois de } 12 \text { meses. }\end{array}$ \\
\hline
\end{tabular}

\begin{tabular}{ll}
\hline $\begin{array}{l}\text { 5. The Moderating } \\
\text { Role of } \\
\text { Different Sources } \\
\text { of Perceived Social }\end{array}$ & $\begin{array}{l}\text { Coeficiente de } \\
\text { correlação de } \\
\text { Pupport on the }\end{array}$ \\
$\begin{array}{l}\text { Pispositional } \\
\text { Optimism- }\end{array}$ & $\begin{array}{l}\text { Regressões } \\
\text { Hierárquicas }\end{array}$ \\
$\begin{array}{l}\text { Posttraumatic } \\
\text { Growth }\end{array}$ & (2 steps) \\
Relationship in & \\
Postoperative & \\
Breast & \\
Cancer Patients & \\
\end{tabular}

Os resultados indicaram que mulheres sobreviventes de câncer de mama com altos níveis de otimismo e suporte social percebido estão mais predispostas a desenvolver crescimento pós-traumático (PTG). Dentre os diferentes tipos de suporte social, apenas o suporte social recebido de uma pessoa íntima foi moderador de otimismo e crescimento pós-traumático.

As três hipóteses testadas foram confirmadas:

I. mulheres no pós-operatório de câncer de mama que são altas em otimismo seriam mais propensas a desenvolver PTG;

2. mulheres no pós-operatório de câncer de mama que são altas em suporte social percebido seriam mais propensas a desenvolver PTG; 3. mulheres no pós-operatório de câncer de mama que são baixas em otimismo seriam mais propensas a não desenvolver PTG mesmo diante de suporte social percebido de uma pessoa íntima.

\begin{tabular}{|c|c|c|}
\hline $\begin{array}{l}\text { 6. Relationships } \\
\text { among optimism, } \\
\text { well-being, self- } \\
\text { transcendence, } \\
\text { coping, and social } \\
\text { support in women } \\
\text { during treatment } \\
\text { for breast cancer }\end{array}$ & $\begin{array}{l}\text { Coeficiente de } \\
\text { correlação de } \\
\text { Pearson } \\
\text { Regressões } \\
\text { Hierárquicas } \\
\text { Múltiplas } \\
\text { (2 steps) }\end{array}$ & $\begin{array}{l}\text { Durante o tratamento de câncer de mama, os efeitos positivos } \\
\text { do otimismo sobre o bem-estar subjetivo são em parte mediados pelo } \\
\text { grau de autotranscedência da mulher. Uma breve triagem do otimismo } \\
\text { das mulheres pode ajudar a identificar mulheres em risco de sofrimento } \\
\text { psíquico. A detecção precoce e a intervenções para promover } \\
\text { o ajustamento psicológico em toda a trajetória do câncer (por exemplo, } \\
\text { aumentar a autotranscedência) devem receber atenção em pesquisas } \\
\text { futuras. }\end{array}$ \\
\hline $\begin{array}{l}\text { 7. Brief Report: } \\
\text { Correlates of Quality } \\
\text { of Life-related } \\
\text { Outcomes in Breast } \\
\text { Cancer Patients } \\
\text { Participating in the } \\
\text { Pathfinders Pilot } \\
\text { Study }\end{array}$ & $\begin{array}{l}\text { Coeficiente de } \\
\text { correlação de } \\
\text { Spearman }\end{array}$ & $\begin{array}{l}\text { Neste estudo piloto, as mudanças nos alvos predefinidos no Programa } \\
\text { Pathfinders, como habilidades de enfrentamento, suporte social, } \\
\text { autoeficácia, espiritualidade e otimismo, correlacionaram-se com } \\
\text { melhorias nos resultados relatados pelo paciente. Este estudo piloto } \\
\text { fornece a confirmação inicial de que os participantes do Programa } \\
\text { Pathfinder experimentaram mudanças nos recursos direcionados, que se } \\
\text { correlacionou com mudanças positivas nos resultados do tratamento. } \\
\text { A pesquisa atual fornece evidências da viabilidade do programa Pathfinder } \\
\text { e do modelo conceitual para a intervenção e avaliação do seu impacto. }\end{array}$ \\
\hline
\end{tabular}

Fonte: Elaborado pelos autores. 


\section{Discussão}

O objetivo deste estudo foi sintetizar as evidências disponíveis quanto à relação entre otimismo e suporte social em mulheres com câncer de mama. Podem-se citar como principais achados: 1 . o corpo de conhecimento disponível confirma a associação entre otimismo e suporte social em mulheres com CA de mama, porém, não é possível estabelecer relações causais entre essas variáveis por causa do delineamento transversal adotado nos estudos; 2 . o estudo da associação entre otimismo e suporte social na área da saúde e psicologia é recente, sendo que a maioria dos estudos foi desenvolvida nos últimos seis anos; 3 . nenhum estudo sobre a associação dessas variáveis tendo como amostra mulheres com CA de mama foi conduzido no Brasil.

Os estudos demonstram que as mulheres podem contar com vários recursos internos e externos para lidar com sua doença, como o otimismo (Carver et al., 1993; Epping-Jordan et al., 1999) e o suporte social (Cohen \& Syme, 1985; Glanz \& Lerman, 1992). A evidência da associação entre essas variáveis sugere que a presença tanto do otimismo quanto do suporte social reduz significativamente respostas de sofrimento emocional diante do diagnóstico e tratamento do câncer, bem como favorece maiores cuidados com a saúde (Wimberling, Carver, \& Antoni, 2008).

$O$ delineamento transversal, presente em quase a totalidade dos estudos aqui apresentados, impossibilita avançar na análise quanto aos fatores determinantes do otimismo e suporte social. Contudo, as evidências geradas podem apontar uma tendência de associação, gerar novas perguntas de pesquisa e dar sustentação para o desenvolvimento de estudos longitudinais que permitam identificar a extensão do impacto do otimismo e do suporte social a longo prazo em mulheres com CA de mama. Isso seria importante já que vários resultados identificaram a significativa relação entre essas variáveis, seus papéis de mediação e moderação para o bem-estar, enfrentamento, coping e redução de sofrimento emocional nos diferentes estágios do CA de mama e seu tratamento.

Outro ponto interessante são os instrumentos usados para medir otimismo e suporte social. Todos os estudos utilizaram a escala Life Orientation Test Revised (LOT-R) ou sua primeira versão, a LOT, para medir otimismo. Essas escalas foram construídas para avaliar o construto otimismo disposicional. A utilização delas em todos os estudos demonstra a opção teórica da comunidade científica sobre o construto otimismo. Entretanto, o suporte social não apresenta a mesma unidade. Para medi-lo, a maior parte dos estudos utilizou uma escala distinta. O suporte social é um construto complexo e multidimensional, e diversas teorias procuram explicá-lo. Isso justifica o grande número de instrumentos existentes. Boa parte dos diversos instrumentos que existem capturam parte ou determinadas nuanças do construto, por isso, a escolha do pesquisador por determinada escala deve estar em consonância com o referencial teórico que embasa o conceito de suporte social. É necessário que o pesquisador tenha um bom embasamento teórico sobre o fenômeno que está investigando e consciência das forças e limitações de o instrumento de medida escolhido. 
Vale destacar, também, que os estudos encontrados nesta revisão foram publicados a partir de 2003, refletindo a atualidade deste enfoque de investigação e o quanto ainda há para ser pesquisado na área. Outro ponto a se considerar é que não foram encontradas pesquisas brasileiras ou latino-americanas sobre as variáveis investigadas no presente estudo. Essas evidências indicam a necessidade e a oportunidade para desenvolvimento da produção científica nacional nesta área.

\section{Considerações finais}

De um modo geral, este artigo possibilitou um entendimento sobre as relações entre otimismo e suporte social em mulheres com câncer de mama. Observa-se que a produção científica sobre essa temática ainda é escassa e apresenta um crescimento lento. Porém, foram encontrados estudos com contribuições interessantes no que diz respeito às interações das variáveis investigadas e suas contribuições para o bem-estar e enfrentamento do câncer de mama. Isso demonstra que esta é uma área promissora para a atuação da psicologia, especialmente no Brasil.

Um dos limitadores deste estudo pode ter sido a restrição das buscas às principais bases de dados em Ciências da Saúde. Desse modo, estudos publicados em periódicos não indexados e relatórios de pesquisa não foram incluídos na revisão. Por ser este um assunto que conta ainda com poucas pesquisas, sugere-se a inclusão de outras fontes de busca em estudos futuros.

Os descritores usados podem ser outro fator limitador. Aqui se apresentam duas questões relevantes para os estudos de revisão sistemática: a indicação dos descritores por parte do pesquisador e a indexação dos documentos nas bases de dados. Por exemplo, foi apenas em 2003 que se introduziu o descritor "psicologia positiva" no Thesaurus da American Psychological Association (APA) e associaram-se temas relevantes a esse campo, como bem-estar, satisfação de vida, otimismo. Em virtude dessa problemática, é possível que muitos estudos não sejam recuperados em uma busca.

Por fim, embora existam as limitações supracitadas, o presente estudo encontrou evidências de correlação positiva entre otimismo e suporte social para o bem-estar das mulheres com câncer de mama, e para o engajamento em ações de cuidado com a saúde física e mental. Diante da carência de estudos, evidenciada pelos resultados desta pesquisa, e sendo o câncer de mama um dos tipos de neoplasia mais frequentes no mundo, o segundo mais comum entre as mulheres, e a terceira causa de morte entre brasileiras adultas (INCA/2014) salienta-se a importância de maior investimento em pesquisas sobre a referida temática.

\section{Referências}

Bozo, Ö., Gündoğdu, E., \& Büyükaşik-Çolak, C. (2009). The moderating role of differentsources of perceived social support on the dispositional optimism-posttraumatic growth relationship in postoperative breast cancer patients. Journal of Health Psychology, 14(7), 1009-1020. DOI: 10.1177/1359105309342295. 
Carver, C. S., Pozo, C., Harris, S. D., Noriega, V., Scheier, M. F., Robinson, D. S., \& Clark, K. C. (1993). How coping mediates the effect of optimism on distress: a study of women with early stage breast cancer. Journal of Personality and Social Psychology, 65(2), 375. DOI: http://dx.doi.org/10.1037/0022-3514.65.2.375.

Cohen, S. E., \& Syme, S. (1985). Social support and health. San Diego: Academic Press.

Epping-Jordan, M. P., Picciotto, M. R., Changeux, J. P., \& Pich, E. M. (1999). Assessment of nicotinic acetylcholine receptor subunit contributions to nicotine self-administration in mutant mice. Psychopharmacology, 147(1), 25-26. DOI: 10.1007/ s002130051135.

Glanz, K., \& Lerman, C. (1992). Psychosocial impact of breast cancer: A critical review. Annals of Behavioral Medicine, 14(3), 204-212.

Instituto Nacional de Câncer José Alencar Gomes da Silva (INCA) (2016). Tipos de câncer - mama. Recuperado em 29 agosto, 2016, de http://www2.inca.gov.br/ wps/wcm/connect/tiposdecancer/site/home+/mama/cancer_mama.

Karademas, E. C. (2006). Self-efficacy, social support and well-being: The mediating role of optimism. Personality and Individual Differences, 40(6), 1281-1290. DOI: 10.1016/j.paid.2005.10.019.

Matthews, E. E., \& Cook, P. F. (2009). Relationships among optimism, well-being, self-transcendence, coping, and social support in women during treatment for breast cancer. Psycho-Oncology, 18(7), 716-726. DOI: 10.1002/pon.1461.

Pacico, J. C., \& Bastianello, M. R. (2014). As origens da psicologia positiva e os primeiros estudos brasileiros. In Hutz, C. S. Avaliação em Psicologia Positiva. Porto Alegre: Artes Médicas.

Pureza, J. D. R., Kuhn, C. H. C., Castro, E. K. D., \& Lisboa, C. S. D. M. (2012). Psicologia positiva no Brasil: uma revisão sistemática da literatura. Revista Brasileira de Terapias Cognitivas, 8(2), 109-117. DOI: 10.5935/1808-5687.20120016.

Rossi, L., \& Santos, M. A. D. (2003). Repercussões psicológicas do adoecimento e tratamento em mulheres acometidas pelo câncer de mama. Psicologia: Ciência e Profissão, 23(4), 32-41.

Scheier, M. F., \& Carver, C. S. (1985). Optimism, coping, and health - assessment and implications of generalized outcome expectancies. Health Psychology, 4(3), 219-247. DOI: http://dx.doi.org/10.1037/0278-6133.4.3.219.

Scheier, M. F., Carver, C. S., \& Bridges, M. W. (1994). Distinguishing optimism from neuroticism (and trait anxiety, self-mastery, and self-esteem): a reevaluation of the life orientation test. Journal of Personality and Social Psychology, 67(6), 1063-1078. DOI: http://dx.doi.org/10.1037/0022-3514.67.6.1063.

Shelby, R. A., Crespin, T. R., Wells-Di Gregorio, S. M., Lamdan, R. M., Siegel, J. E., \& Taylor, K. L. (2008). Optimism, social support, and adjustment in African American women with breast cancer. Journal of Behavioral Medicine, 31(5), 433-444. DOI: $10.1007 / \mathrm{s} 10865$ 008-9167-2. 
Silva, L. C. (2008). Câncer de mama e sofrimento psicológico: aspectos relacionados ao feminino. Psicologia em Estudo, 13(2), 231-237.

Smith, S. K., Herndon, J. E., Lyerly, H. K., Coan, A., Wheeler, J. L., Staley, T., \& Abernethy, P. (2011). Correlates of quality of life-related outcomes in breast cancer patients participating in the pathfinders pilot study. Psycho-Oncology, 20(5), 559-564. DOI: 10.1002/pon.1770.

Symister, P., \& Friend, R. (2003). The influence of social support and problematic support on optimism and depression in chronic illness: a prospective study evaluating self-esteem as a mediator. Health Psychology, 22(2), 123-129. DOI: http:// dx.doi.org/10.1037/0278-6133.22.2.123.

Taylor, S. E., Kemeny, M. E., Aspinwall, L. G., Schneider, S. G., Rodriguez, R., \& Herbert, M. (1992). Optimism, coping, psychological distress, and high-risk sexual behavior among men at risk for acquired immunodeficiency syndrome (AIDS). Journal of Personality and Social Psychology, 63(3), 460.

Trunzo, J. J., \& Pinto, B. M. (2003). Social support as a mediator of optimism and distress in breast cancer survivors. Journal of Consulting and Clinical Psychology, 71(4), 805-811. DOI: http://dx.doi.org/10.1037/0022-006X.71.4.805.

Von Ah, D., Kang, D. H., \& Carpenter, J. S. (2007). Stress, optimism, and social support: Impact on immune responses in breast cancer. Research in Nursing \& Health, 30(1), 72-83. DOI: 10.1002/nur.20164.

Zenger, M., Glaesmer, H., Höckel, M., \& Hinz, A. (2011). Pessimism predicts anxiety, depression and quality of life in female cancer patients. Japanese Journal of Clinical Oncology, 41(1), 87-94. DOI: 10.1093/jjco/hyq168.

Wimberly, S. R., Carver, C. S., \& Antoni, M. H. (2008). Effects of optimism, interpersonal relationships, and distress on psychosexual well-being among women with early stage breast cancer. Psychology and Health, 23(1), 57-72. DOI: 10.1080/14768320701204211.

Submissão: 28.3.2016

Aceitação: 29.4.2016 